\title{
Novel High Quality Data Hiding System
}

\author{
Fahd Alharbi \\ Faculty of Engineering, King Abdulaziz University Rabigh, KSA \\ Fahdalharbi@kau.edu.sa
}

\begin{abstract}
Data Hiding is the process of embedding data into a media form such as image, voice, and video. The major methods used for data hiding are the frequency domain and the spatial domain. In the frequency domain, the secret data bits are inserted into the coefficients of the image pixel's frequency representation such as Discrete Cosine Transform (DCT), Discrete Fourier Transform (DFT) and Discrete Wavelet Transform (DWT). On the other hand, in the spatial domain method, the secret data bits are inserted directly into the images' pixels value decomposition. The Lest Significant Bit (LSB) is consider as the most widely spatial domain method used for data hiding. LSB embeds the secret message's bits into the least significant bit plane( Binary decomposition) of the image in a sequentially manner. The LSB is simple, but it poses some critical issues. The secret message is easily detected and attacked duo to the sequential embedding process. Moreover, embedding using a higher bit plane would degrade the image quality. In this paper, we are proposing a novel data hiding method based on Lucas number system. We use Lucas number system to decompose the images' pixels values to allow using higher bit plane for embedding without degrading the image's quality. Moreover, the data hiding process security is enhanced by using Pseudo Random Number Generators(PRNG) for selecting the image's pixels used for embedding data.
\end{abstract}

Index Terms - Data Hiding, Image, LSB, Lucas, PRNG

\section{INTRODUCTION}

The goal of the data hiding system is to communicate a secret message in a way that would not be noticeable by an intruder [1-3]. There are two techniques are regularly used for data hiding, the frequency domain[45] and the spatial domain [6-10]. In the frequency domain data hiding techniques, the secret data bits are inserted into the coefficients of the image pixel's frequency representation. Among the frequency image pixel's frequency representation are Discrete Cosine Transform (DCT) [11], Discrete Fourier Transform (DFT) [12] and Discrete Wavelet Transform (DWT) [13]. On the other hand, in the spatial domain techniques, the secret data bits are inserted directly into the images' pixels value decomposition. In this section, we are discussing the Lest Significant Bit (LSB) which is consider as the most widely spatial domain method used for data hiding

The least significant bit (LSB) data hiding technique [6-10] is the process of inserting the secret message's data bits into the least significant bits of the image (Binary Form) in a sequential manner [14-17]. For illustration, let I be the original gray scale image where each pixel is represented using 8-bit format, thus each pixel's value is varies from 0 up to 255 as illustrated at Table 1

Table 1. Original IMAge PiXels (BINARy)

\begin{tabular}{|c|c|}
\hline \multicolumn{2}{|c|}{ Pixels before embedding } \\
\hline 1st & 01001100 \\
\hline 2nd & 01001101 \\
\hline 3rd & 01001110 \\
\hline 4th & 01001111 \\
\hline 5th & 01010000 \\
\hline 6th & 01001011 \\
\hline 7th & 01010001 \\
\hline 8th & 01010001 \\
\hline
\end{tabular}

The secret message is simply the letter $\mathrm{Z}$ with its binary representation as 01011010. The LSB embedding process is shown at Table 2 , where 8 pixels are used to hide the letter $Z$. The secret message's bits are inserted into the least significant bit of the image's pixels in a sequential manner. The LSB data hiding technique is simple and the effect on the image quality is limited and hardly noticed by the human eye duo to the small value of the bit (least significant bit) used for embedding. On the other hand, the LSB is easy to be detected and attacked by simply extracting or changing the least significant bits of each pixel.

TABLE 2. LSB EMBEDDING

\begin{tabular}{|c|c|}
\hline $\begin{array}{c}\text { Secret bits } \\
(\mathrm{Z})\end{array}$ & $\begin{array}{c}\text { Pixels after } \\
\text { embedding }\end{array}$ \\
\hline 0 & $0100110 \underline{0}$ \\
\hline 1 & $0100110 \underline{1}$ \\
\hline 0 & $0100111 \underline{0}$ \\
\hline 1 & $0100111 \underline{1}$ \\
\hline 1 & $0101000 \underline{1}$ \\
\hline 0 & $0100101 \underline{0}$ \\
\hline 1 & $0101000 \underline{1}$ \\
\hline 0 & $0101000 \underline{0}$ \\
\hline
\end{tabular}

On the other hand, using higher bits for embedding the secret message would enhance the security and at the same time degrade the image quality. For example, as 
illustrated at Table 3; using the fifth bit for hiding the secret message would degrade the image quality duo to the fact that the value of the fifth bit is 16 and the impact would be clear.

TABLE 3. LSB EMBEDDING ( USING FIFTH BIT)

\begin{tabular}{|c|c|c|}
\hline $\begin{array}{c}\text { Secret bits } \\
(\mathrm{Z})\end{array}$ & $\begin{array}{c}\text { Pixels after } \\
\text { embedding }\end{array}$ & difference \\
\hline 0 & $010 \underline{01} 100$ & 0 \\
\hline 1 & $010 \underline{11} 1101$ & 16 \\
\hline 0 & $010 \underline{01110}$ & 0 \\
\hline 1 & $010 \underline{11111}$ & 16 \\
\hline 1 & $010 \underline{10000}$ & 0 \\
\hline 0 & $010 \underline{0} 1011$ & 0 \\
\hline 1 & $010 \underline{10} 001$ & 0 \\
\hline 0 & $010 \underline{00001}$ & -16 \\
\hline
\end{tabular}

In this paper, we propose using Lucas number system for image pixel's value decomposition to allow using higher bit plane without degrading the image quality. Also, we are enhancing the security of the data hiding system by using Pseudo Random Number Generator to select the next pixel used for embedding. The rest of the paper is organized as follows: Section II discusses the Lucas based hiding system; Section III describes enhancing the data hiding system's security by using Pseudo Random Number Generators to select the next pixel for embedding; Section IV presents experimental results; we finally conclude in Section V.

\section{LUCAS BASED HIDING SYSTEM}

Now, we are proposing using the Lucas numbers [1819] for pixels values decomposition. The Lucas sequence generated using the following formula

$L_{n}=L_{n-1}+L_{n-2} \quad, n>2$

where, $L_{1}=2$ and $L_{2}=1$

The image's pixels values would be represented as the sum of the non-consecutive Lucas numbers [20-21]. To represent the range of 0 to 255, we need 12-bit of Lucas digits.

$$
\begin{array}{llllllllllll}
L_{12} & L_{11} & L_{10} & L_{9} & L_{8} & L_{7} & L_{6} & L_{5} & L_{4} & L_{3} & L_{2} & L_{1}
\end{array}
$$$$
\begin{array}{llllllllllll}
199 & 123 & 76 & 47 & 29 & 18 & 11 & 7 & 4 & 3 & 1 & 2
\end{array}
$$

Now, we represent the pixel value of 26 using Binary and Lucas representation as follows

The binary representation is $\quad 00011010$

The Lucas representation is $\quad 000001010010$

Let consider hiding the bit value of 0 using the fifth bit in each decomposition of the pixel value of 26 . The result is as following

The binary representation is

$$
000 \underline{0} 1010
$$

The Lucas representation is $0000010 \underline{0} 0010$

The pixel value after LSB embedding using the fifth bit is 10 , while the pixel value after embedding using Lucas decomposition is 19. It is clear that using the
Lucas decomposition would result in higher quality data embedding duo to the fact that the Lucas bits are less significant than those of the Binary decomposition.

Here, we reconsider the example of hiding the letter $\mathrm{Z}$ in eight pixels (Table 1). The eight pixels values in Lucas system are shown at Table 4.

TABLe 4. Original IMAGe PiXels (LuCAS )
\begin{tabular}{|c|c|}
\hline \multicolumn{2}{|c|}{ Pixels before embedding } \\
\hline 1st & 001000000000 \\
\hline 2nd & 001000000010 \\
\hline 3rd & 001000000001 \\
\hline 4th & 001000000100 \\
\hline 5th & 001000001000 \\
\hline 6th & 000101010100 \\
\hline 7th & 001000001010 \\
\hline 8th & 001000001010 \\
\hline
\end{tabular}

Table 5 shows the result of embedding the letter $\mathrm{Z}$ into eight pixels using Lucas decomposition, where the image quality after embedding is better the that achieved using LSB technique duo to the fact that the digits in Lucas system are less significant (7) than those in binary system (16).

TABLE 5. LUCAS EMBEDding ( USING FIFTH BIT)

\begin{tabular}{|c|c|c|}
\hline $\begin{array}{c}\text { Secret } \\
\text { bits }(\mathrm{Z})\end{array}$ & $\begin{array}{c}\text { Pixels after } \\
\text { embedding }\end{array}$ & difference \\
\hline 0 & $0010000 \underline{0} 0000$ & 0 \\
\hline 1 & $0010000 \underline{10010}$ & 7 \\
\hline 0 & $0010000 \underline{0} 0001$ & 0 \\
\hline 1 & $0010000 \underline{10100}$ & 7 \\
\hline 1 & $0010000 \underline{10000}$ & 3 \\
\hline 0 & $0001010 \underline{0} 0100$ & -7 \\
\hline 1 & $0010000 \underline{10010}$ & 3 \\
\hline 0 & $0010000 \underline{0} 1010$ & 0 \\
\hline
\end{tabular}

\section{PSEUdo RANDOM NUMBER GENERATOR}

The LSB technique is simple but not secure. The intruder can easily recovered the hidden message by extracting the least significant bits. On the other hand, using higher bits for embedding would degrade the image quality. In this section, we enhance the data hiding system's security by using Pseudo Random Number Generators to select the next pixel for embedding. The Pseudo Random Sequence is generated using Non-Linear forward feedback shift Register (NLFFSR) [22-23]. To start generating the Pseudo Random Sequence, the registers simply loaded with any initial value except zero and with each clock (step) a new Random Number is generated. The feedback function of the Pseudo Random Sequence Generator is designed based on the characteristic polynomial of the Generator. The characteristic polynomial is in the form of

$$
P(x)=x^{n}+a_{n-1} x^{n-1} \ldots \ldots+a_{1} x+a_{0}
$$


where, $\mathrm{n}$ represents the number of registers and the length of the generated sequence is

$$
N=2^{n}-1
$$

Let consider an image $I_{R, C}$, where $\mathrm{C}$ is the number of columns and $\mathrm{R}$ is the number of pixels (rows) in each columns. To increase the security of the data hiding system, we use three Random Sequence Generators to select the next pixel and bit plane used for embedding $I_{i, j}^{b}$. The first Generator selects a column $j$ in the image, the second Generator selects a pixel (row) $i$ at the selected column and the third Generator selects the bit plane ( $b=1,2 \ldots . .8$ ) used for embedding. Moreover, we may use two Random Sequence Generators to select the byte $L$ of the secret data and the bit $K$ to be embedded, $D_{L}^{K}$. The secure data hiding system may uses up to six Random Sequence Generators to perform the data embedding process as the following

$$
I_{i, j}^{b}=D_{L}^{k}
$$

Now, we enhancing the embedding of the letter $\mathrm{Z}$ into the eight pixels by using Random Sequence Generators. Let assume that the coulomb $j$ has been selected and it is contain the pixels values in the sequence shown at Table 1 and the sequence of the rows $i$ (pixel) are in the following random sequence $(6,8,3,5,2,4,7,1)$. The embedding process is shown at Table 6 , where both the image quality and the data security are enhanced.

TABLE 6. LuCAS EMBEDDING + PRNG ( USING FIFTH BIT)

\begin{tabular}{|c|c|c|}
\hline $\begin{array}{c}\text { Secret } \\
\text { bits }(\mathrm{Z})\end{array}$ & $\begin{array}{c}\text { Pixels after } \\
\text { embedding }\end{array}$ & difference \\
\hline 0 & $0001010 \underline{00100}$ & -7 \\
\hline 1 & $0010000 \underline{10010}$ & 3 \\
\hline 0 & $0010000 \underline{00001}$ & 0 \\
\hline 1 & $0010000 \underline{10000}$ & 3 \\
\hline 1 & $0010000 \underline{10010}$ & 7 \\
\hline 0 & $0010000 \underline{0} 0100$ & 0 \\
\hline 1 & $0010000 \underline{10010}$ & 3 \\
\hline 0 & $0010000 \underline{00000}$ & 0 \\
\hline
\end{tabular}

\section{EXPERIMENTAL RESULTS}

The quality of the embedding techniques are evaluated by the Peak Signal to Noise Ratio ( PSNR), where it is defined as

$P S N R=10 \log _{10}\left(\frac{I_{M A X}^{2}}{M S E}\right)$

where, $I_{M A X}^{2}$ is the maximum possible value of a pixel.

In this experiment (gray scale image), $I_{M A X}^{2}$ is equal to 255. The Mean Square Error (MSE) is computed as follows
$M S E=\frac{1}{R \times C} \sum_{i=1}^{R} \sum_{j=1}^{C}\left(\left|I_{i, j}-I_{i, j}^{\prime}\right|\right)^{2}$

Where, $I_{i, j}$ is the original image's pixel value and $I_{i, j}^{\prime}$ is the image's pixel value after embedding.

In this section, we first evaluate the performance of the data hiding techniques using different bit planes for embedding the secret message's bits. For data hiding, we are using a gray scale $512 \times 512$ pixels image (Figure 1). The hiding capacity of the original image is 32768 data bytes, where each pixel is used for hiding a single data bit. We vary the bit plane used for embedding from the first bit up to the eighth bit in each data hiding technique. In each case we hide 32768 data bytes and evaluate the performance by computing the PSNR.

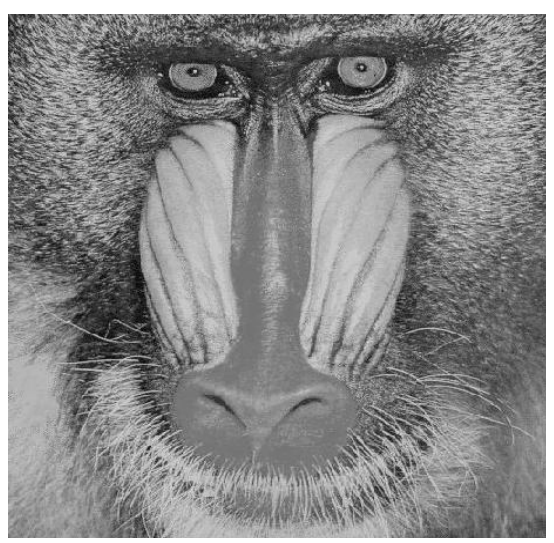

Figure 1.Original Image

The performance of the Embedding Techniques is shown at Figures(2-9). The higher the bit plane used for embedding the higher the impact of the hiding process on the covered image quality. The quality of the covered image using the LSB hiding technique is degrade using higher bit plane for embedding. On the other hand, Lucas hiding technique maintains a better image quality for all cases. Moreover, Table 7 shows that Lucas hiding technique outperforms the LSB in achieving better Signal to Noise Ratio (PSNR ) duo to the fact that digits in Lucas number system are less significant than those in binary system. Conversely, the first bit in the binary system is less significant than that of the Lucas number system, thus the LSB achieves better PSNR than the Lucas hiding technique with using the first bit for imbedding which is consider the least security bit plane for data hiding. 


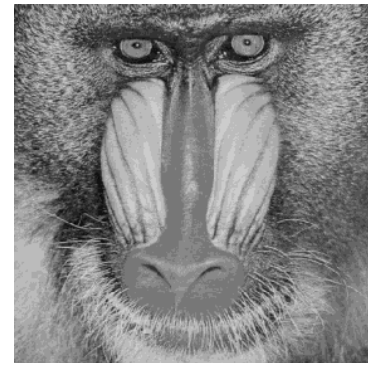

LSB

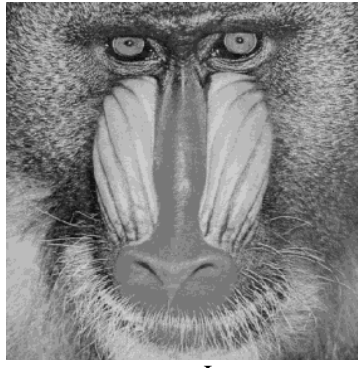

LUCAS
Figure 2. Embedding Techniques Performance ( 1st bit)

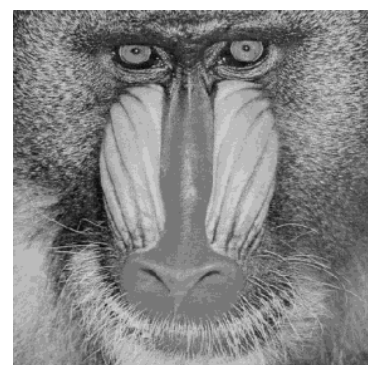

LSB

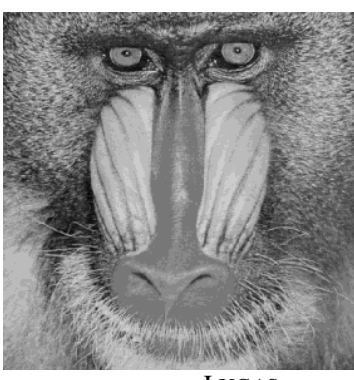

LUCAS
Figure 3. Embedding Techniques Performance ( 2nd bit)

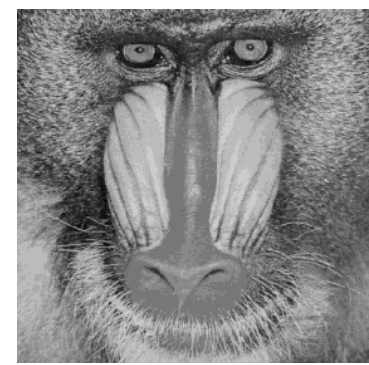

LSB

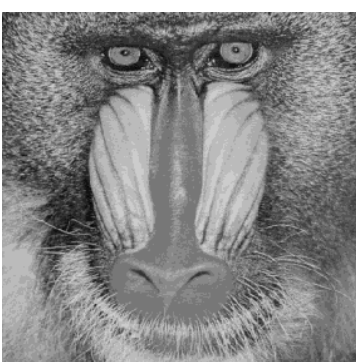

LUCAS
Figure 4. Embedding Techniques Performance ( $3 r d$ bit)

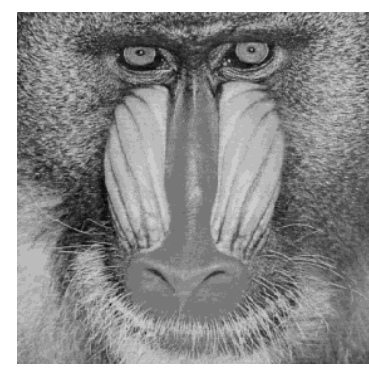

LSB

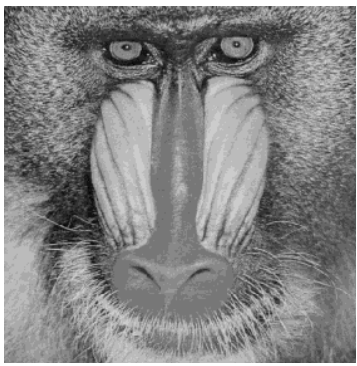

LUCAS
Figure 5. Embedding Techniques Performance (4th bit)

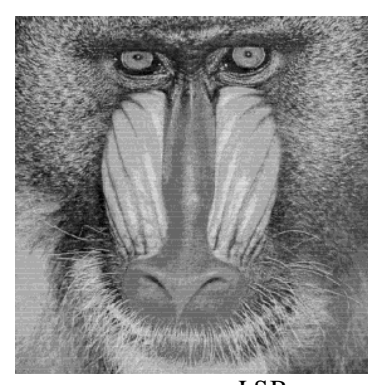

LSB

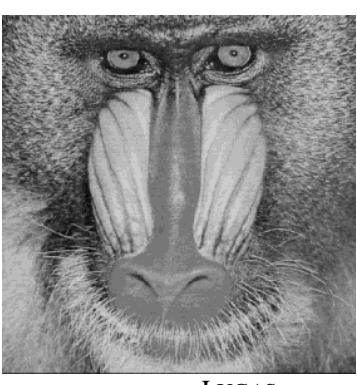

LUCAS
Figure 6. Embedding Techniques Performance ( 5 th bit)

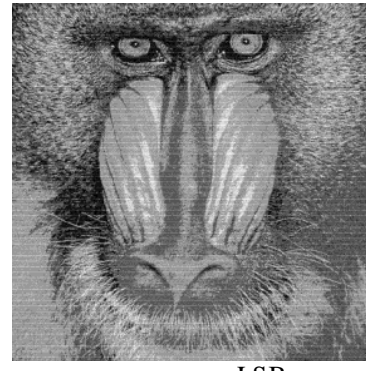

LSB

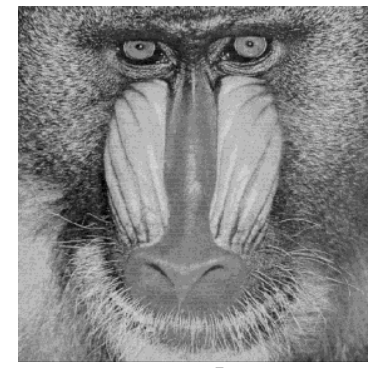

LUCAS

Figure 7. Embedding Techniques Performance ( 6 th bit)

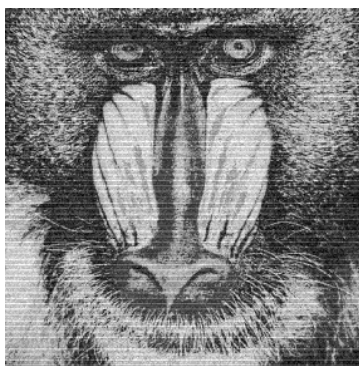

LSB

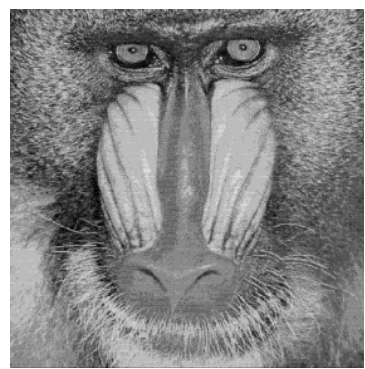

LUCAS

Figure 8. Embedding Techniques Performance ( 7 th bit)
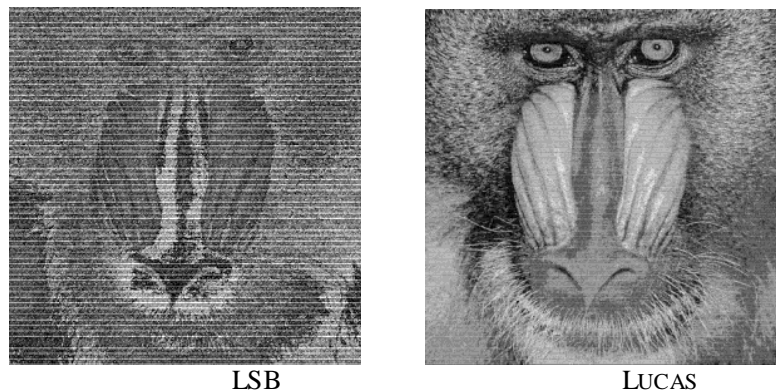

LUCAS

Figure 9. Embedding Techniques Performance ( 8th bit)

Table 7. Embedding Techniques Performance ( PSNR)

\begin{tabular}{|c|c|c|}
\hline \multirow{2}{*}{$\begin{array}{c}\text { Bit } \\
\text { plane }\end{array}$} & \multicolumn{2}{|c|}{ Peak Signal to Noise Ratio ( PSNR) } \\
\cline { 2 - 3 } & LSB & Lucas \\
\hline 1st & 77.0399 & 54.6944 \\
\hline 2nd & 50.7404 & 64.1260 \\
\hline 3rd & 41.7281 & 52.1163 \\
\hline 4th & 35.4584 & 43.6703 \\
\hline 5th & 27.3609 & 38.8316 \\
\hline 6th & 20.522 & 33.4006 \\
\hline 7th & 14.9971 & 27.7555 \\
\hline 8th & 8.9157 & 22.72 \\
\hline
\end{tabular}

In the second experiment, we are using a gray scale $256 \times 256$ pixels image (Figure 10 ). The hiding capacity of the original image is 8192 data bytes, where each pixel is used for hiding a single data bit. The embedding process ( Eq. 4) is performed using five Pseudo Random Sequence Generators. The first Generator selects a secret data byte $L$ and the second Generator picks the bit $K$ to be embedded in the following sequence ( $K=5,7,8,1,2,4,3,6)$. The third and the fourth Generators select the position (column $j \&$ row $i$ ) of 
the pixel to be used for embedding as shown at Figures(11-12). Finally, the fifth Generator selects the bit plane ( $b=4,3,6,5,7,8,1,2)$ used for data hiding. We vary the size of the secret data from $10 \%$ of the hiding capacity up to $100 \%$ of the hiding capacity and in each case we evaluate the performance of the hiding techniques by computing the PSNR. The performance of the LSB Embedding Technique is shown at Figures(1322). The higher the size of the secret data the higher the impact on the covered image quality. On the other hand, the data hiding system using Lucas numbers maintained a better image quality as illustrated at Figure 23. Moreover, the data security is enhanced by using five Pseudo Random Sequence Generators.

\section{Conclusions}

The main objective of the data hiding system is to communicate a secret message in a way that would not be noticeable by an intruder. The least significant bit (LSB) is the most widely used technique for data hiding. The LSB process is simple but not secure. Also, using higher bit plane for hiding data would degrade the covered image's quality. In this paper, we are proposing a novel data hiding method based on Lucas number system. We use Lucas number system to decompose the images' pixels values to allow using higher bit plane for embedding without degrading the image's quality. Also, we enhanced the data security by using Pseudo Random Number Generators(PRNG). The performance of the LSB and the proposed method are evaluated by computing the Peak Signal to Noise Ratio (PSNR), where the proposed method achieved better performance than the LSB.

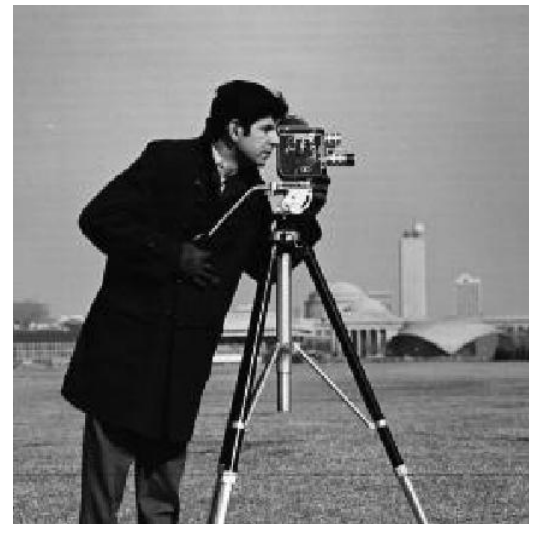

Figure 10. cameraman Image

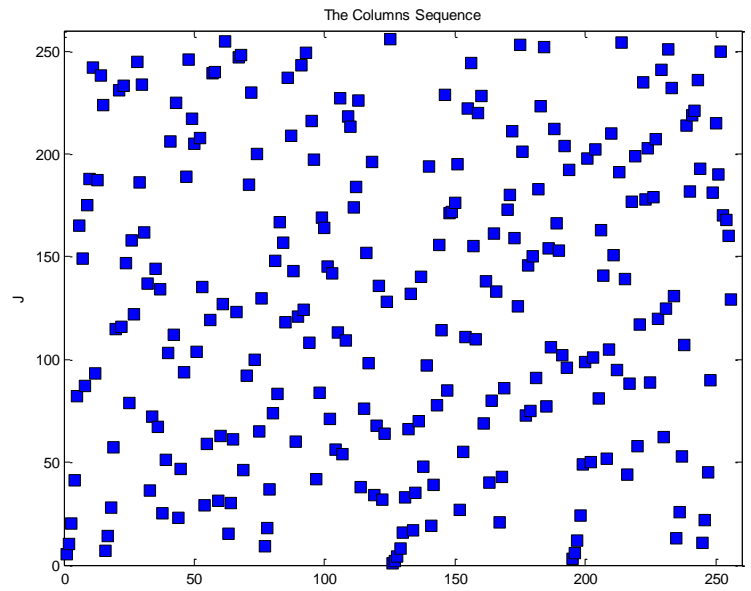

Figure 11. The third generator

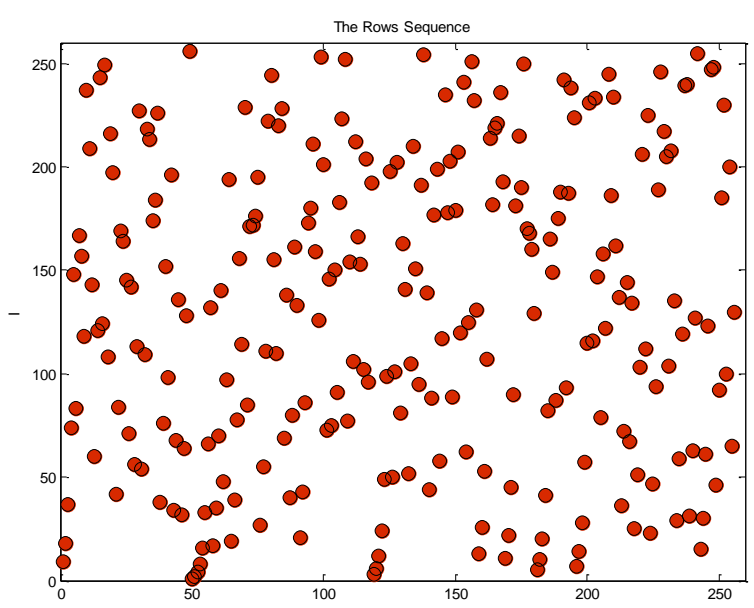

Figure 12. The fourth Generator
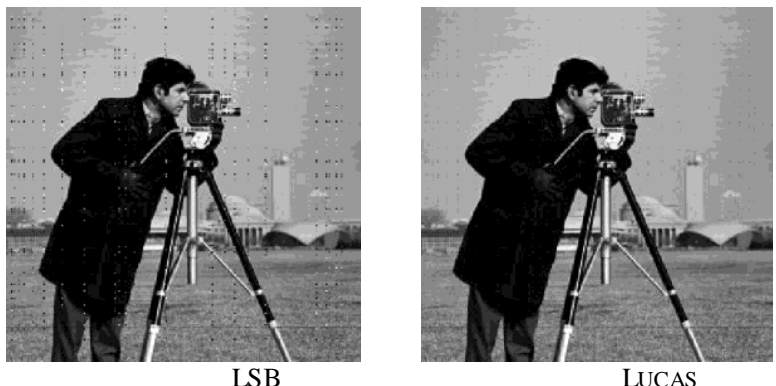

Figure 13. Techniques Performance (10\% of hiding capacity)
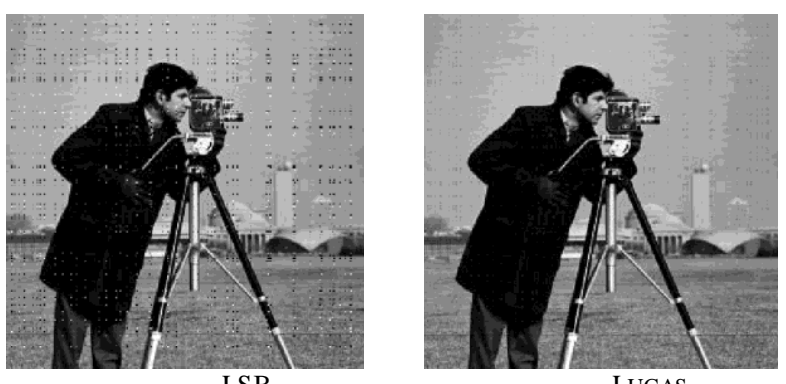

LSB

LUCAS

Figure 14. Techniques Performance (20\% of hiding capacity) 


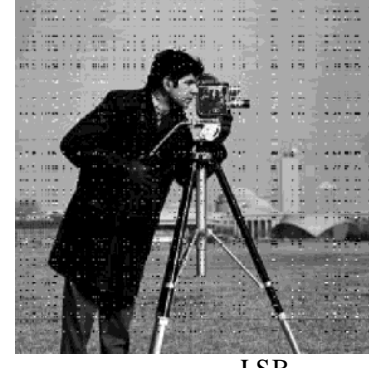

LSB

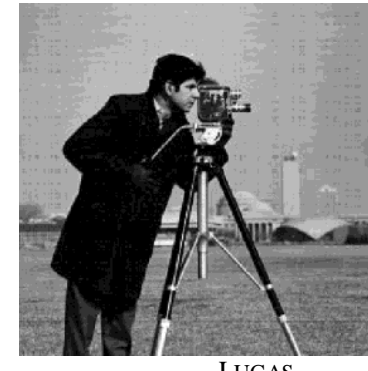

LUCAS
Figure 15 . Techniques Performance (30\% of hiding capacity)

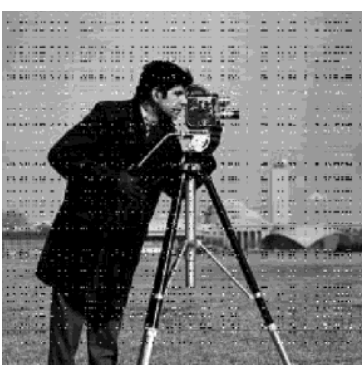

LSB

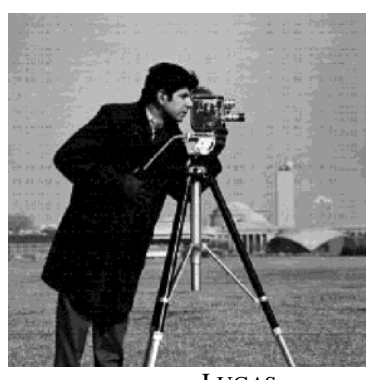

LUCAS
Figure 16. Techniques Performance (40\% of hiding capacity)

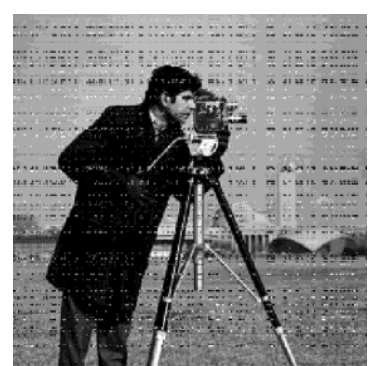

LSB

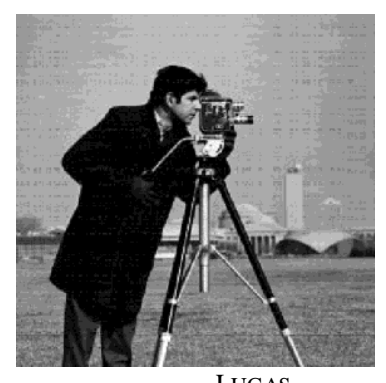

LUCAS
Figure 17. Techniques Performance (50\% of hiding capacity)

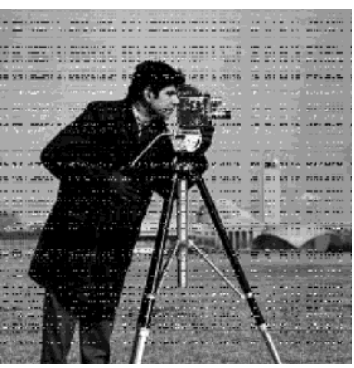

LSB

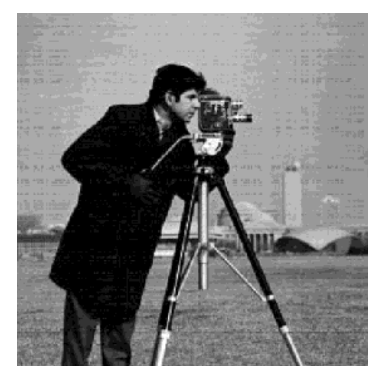

LUCAS
Figure 18 . Techniques Performance ( $60 \%$ of hiding capacity)

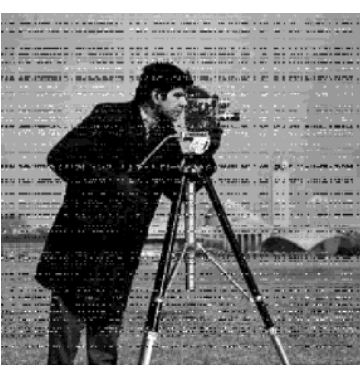

LSB

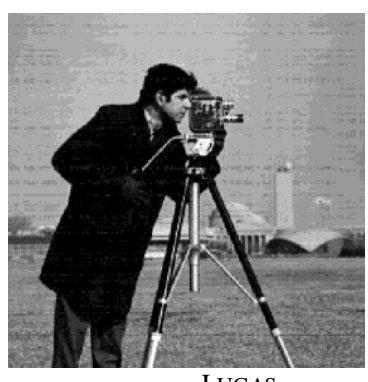

LUCAS
Figure 19. Techniques Performance (70\% of hiding capacity)

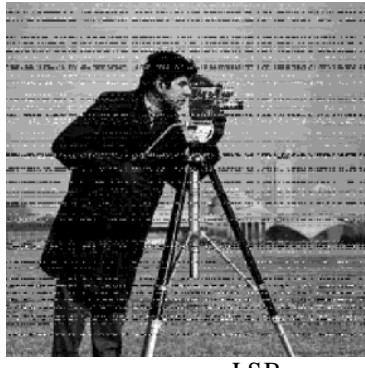

LSB

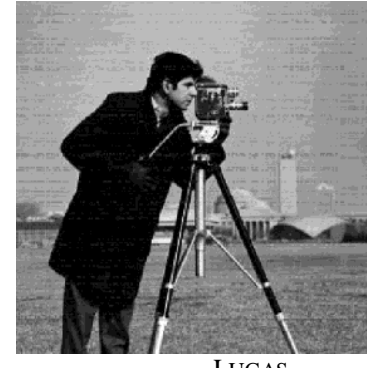

LUCAS
Figure 20. Techniques Performance ( $80 \%$ of hiding capacity)

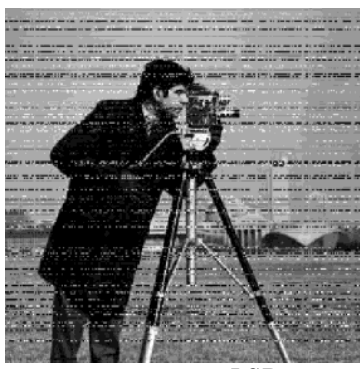

LSB

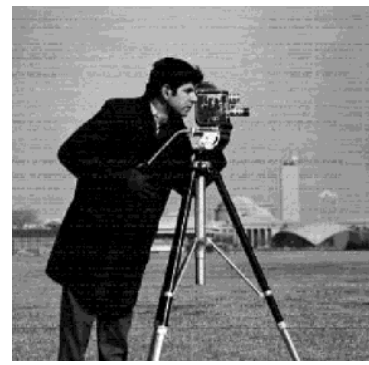

LUCAS
Figure 21. Techniques Performance (90\% of hiding capacity)

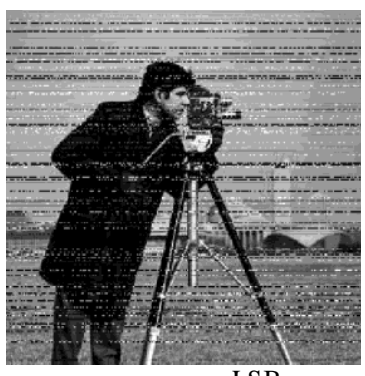

LSB

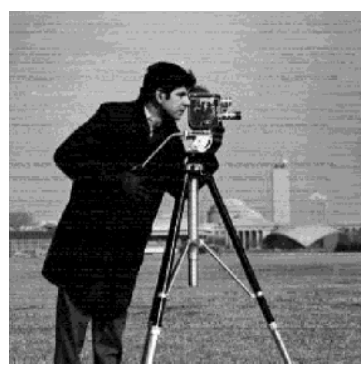

LUCAS
Figure 22. Techniques Performance (100\% of hiding capacity)

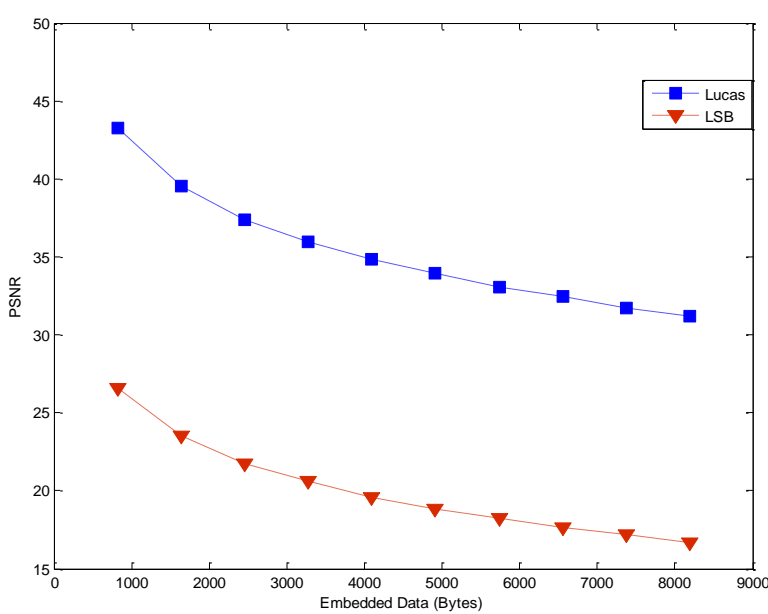

Figure 23. Embedding Techniques Performance ( PSNR)

\section{REFERENCES}

[1] S. Katzenbeisser, F.A.P. Petitcolas, Information Hiding Techniques for Steganography and Digital Watermarking, Artech House, Norwood, MA, 2000.

[2] W. Bender, D. Gruhl, N. Morimoto, A. $\mathrm{Lu},-$ Techniques for data hiding || IBM Syst. J. 35 (3\&4) (1996) 313-336. 
[3] Cox, I., Miller, M., Bloom, J., Fridrich, J., Kalker, T.: Digital Watermarking and Steganography, $2 \mathrm{Nd}$ Ed. ISBN: 978-0123725851

[4] Guorong Xuan, Yun Q. Shi, Zhicheng Ni, "Reversible data hiding using integer wavelet transform and companding technique," IWDW04, Korea, October 2004.

[5] Mauro Barni, Franco Bartolini, Vito Cappellini,Alessandro Piva(1998), "A DCT-domain system for robust image watermarking”, Signal Processing, Vol. 66 (1998), pp.357-372.

[6] Jessica Fridrich and Miroslav Goljan, "On estimation of secret message length in LSB steganography in spatial domain," in Security, Steganography, and Watermarking of Multimedia Contents VI, Proceedings of SPIE 5306, pp. 23-34, 2004.

[7] Y. Qiudong, X. Liu, "A new LSB matching steganographic method based on steganographic information table", IEEE International Conference on Intelligent Networks and Intelligent Systems, pp. 362-365, 2009.

[8] S.M.M. Karim, M.S. Rahman, M.I. Hossain, "A new approach for LSB based image steganography using secret key", IEEE International Conference on Computer and Information Technology, pp. 286191, 2011.

[9] C.H. Yang, C.Y. Weng, S.J. Wang, H.M. Sun, "Adaptive data hiding in edge areas of images with spatial LSB domain systems", IEEE Transactions on Information Forensics and Security, Vol. 3, pp. 488-497, 2008.

[10] K. Ghazanfari, S. Ghaemmaghami, S.R. Khosravi, "LSB++: An improvement to LSB+ steganography", IEEE Region 10 Conference: Tencon 2011, pp. 364-368,2011.

[11] J. R. Hernandez, J. M. Rodr'iguez, and F. P'erezGonz'alez, "Improving the performance of spatial watermarking of images using channel coding," Signal Process. 80(7), pp. 1261-1279, 2000.

[12] Nabin Ghoshal , Jyostna Kumar Mandal, “A Novel Technique for Image Authentication in Frequency Domain using Discrete Fourier Transformation Technique", Malaysian Journal of Computer Science, ISSN 0127-9094, Vol. 21, No. 1, pp. 24-32, 2008.

[13]F. Battisti, K. Egiazarian, M. Carli, and A.Neri, "Data hiding based on Fibonacci-Haar transform," in Mobile Multimedia/Image Processing for Military and Security Applications, SPIE Defense and Security, Vol. 6579, May 2007.

[14] Chi-Kwong Chan and L. M. Cheng, "Hiding data in images by simple LSB substitution," Pattern Recognition, pp. 469-474, Mar. 2004.

[15] Cox, I., Miller, M., Bloom, J., Fridrich, J., Kalker, T.: Digital Watermarking and Steganography, $2 \mathrm{Nd}$ Ed. ISBN: 978-0123725851

[16] Swanson, M. D., Kobayashi, M., Tewfik, A. H.: Multimedia Data-Embedding and watermarking
Technologies, Proc. IEEE, vol. 86, 1064 - 1087, 1998

[17]N. Johnson, Digital Watermarking and Steganography: Fundamentals and Techniques, The Computer Journal. (2009)

[18] G P. Ribenboim, My Numbers, My Friends, Springer, 2000, ISBN 0-38798911-0

[19] L. E. Dickson, "Recurring Series; Lucas' Un, Vn," History of the Theory of Numbers: Divisibility and Primality, Dover Publications, New York, Vol. 1, 2005, pp. 393-411.

[20] Brown, J. L. Jr. "Zeckendorfs Theorem and Some Applications", Fib. Quart. 2, 16 3-168, 1964.

[21]Phillips G.M., "Zeckendorf representation", in Hazewinkel, Michiel, Encyclopaedia of Mathematics, Springer, ISBN 978-1556080104, Picione, 2001.

[22] L. T. Wang and E. J. McCluskey, "Linear feedback shift register design using cyclic codes," IEEE Trans. Comput., vol. 37, pp. 1302-1306, Oct. 1988.

[23] A. Fuster and L. J. Garcia, "An efficient algorithm to generate binary sequences for cryptographic purposes," Theoretical Computer Science, vol. 259, pp. 679-688, May 2001.

Fahd Alharbi is an Assistant Professor, Department of Electrical Engineering, Faculty of Engineering, King Abdulaziz University, Rabigh, KSA. His research interests include computer networks and data communication and security. 